\title{
Interview de Olga BALTAG par Hélène Lenz
}

\section{Olga Baltag et Hélène Lenz}

\section{(2) OpenEdition \\ Journals}

Édition électronique

URL : https://journals.openedition.org/cher/10658

DOI : $10.4000 /$ cher.10658

ISSN : 2803-5992

\section{Éditeur}

Presses universitaires de Strasbourg

\section{Édition imprimée}

Date de publication : 1 décembre 2013

Pagination : 305-313

ISBN : 978-2-86820-560-5

ISSN : 1968-035X

\section{Référence électronique}

Olga Baltag et Hélène Lenz, «Interview de Olga BaLtag par Hélène Lenz », reCHERches [En ligne], 11 | 2013, mis en ligne le 08 février 2022, consulté le 09 février 2022. URL : http://

journals.openedition.org/cher/10658; DOI : https://doi.org/10.4000/cher.10658

\section{(c) (i) (9)}

Ce(tte) œuvre est mise à disposition selon les termes de la Licence Creative Commons Attribution -

Pas d'Utilisation Commerciale - Partage dans les Mêmes Conditions 4.0 International. 


\section{Interview de Olga BaLtag par Hélène Lenz}

\section{Mardi 2 octobre 2012}

Hélène Lenz: Écrivez-vous depuis longtemps? Accepteriez-vous d'évoquer votre milieu familial?

Olga Baltag: J'écris régulièrement depuis 2009. Les premiers poèmes de mon livre Autopsia sufletului/ L'autopsie de l'âme ont été écrits en 2009. En 2009 je présentais mon bac en Moldavie, je savais déjà que j'irais faire des études à Strasbourg en Arts du Spectacle, je les ai commencées en décembre de la même année. Pourquoi avoir choisi la France? Parce que ma mère est professeur de français et mon père ingénieur en construction civile. Ma grand-mère maternelle était aussi professeur de français; elle correspondait avec des Français même si elle n'est jamais venue en France. Avant de passer ses premières vacances de Noël ici, ma mère non plus n'était jamais venue en France. C'était donc un peu aussi la réalisation des rêves de ma grand-mère et de ma mère. Et puisque je parlais le français, je n'ai pas eu vraiment de difficultés au niveau de la langue. Ce sont mes parents qui me prennent en charge financièrement car étudier me prend deux fois plus de temps puisqu'on nous rappelle à l'Université des choses que les lycéens français savent mais que moi, je dois apprendre depuis le début. De même mon frère fait des études à l'étranger, à Dresde: un master de direction d'Orchestre Symphonique. Il a déjà obtenu des prix de Direction à des concours. Il pratique le piano et l'accordéon. Il a 24 ans et s'appelle Roman Baltag. Je crois que le fait d'avoir choisi de faire du théâtre, de me consacrer à l'art est lié à notre éducation dans une atmosphère artistique. Ma mère joue de l'accordéon et du piano, elle a donné des cours de musique au niveau scolaire. À l'âge de 4 ou 5 ans, je savais déjà chanter en français. 
H.L.: Connaissez-vous des gens de votre âge qui écrivent de la poésie, qui la publie?

O. B. : En Moldavie: non, en France: non.

H.L.: Votre orientation vers les arts du spectacle a-t-elle été encouragée par votre famille, par votre cursus scolaire? Qu'attendiez-vous d'une formation en France? Après trois ans d'études en arts du spectacle, pouvez-vous faire un premier bilan d'un changement dans vos goûts initiaux ou au contraire de nouvelles directions possibles?

O. B. : Avant d'arriver à Strasbourg, je voulais être comédienne, d'ailleurs j'ai déjà été comédienne amateur. En Moldavie, j'ai effectué ma scolarité dans un lycée théâtral: "Liceul Teoretic cu profil de arte Elena Alistar». Pour y entrer, j'avais réussi un concours à l'âge de 10 ans. Les épreuves portaient sur la récitation d'une poésie, il fallait aussi connaître une fable, improviser de petites scènes, des situations, faire preuve d'oreille musicale. Le baccalauréat en art que j'ai réussi est un baccalauréat général. Les matières au programme portaient entre autres sur l'histoire du théâtre, la pratique théâtrale, la langue roumaine, les autres matières générales, la connaissance obligatoire d'une langue étrangère: français ou anglais. Assez vite après mon arrivée ici, je me suis rendue compte qu'à l'Université, on ne fait pas de pratique théâtrale. Pour cela, il faut s'inscrire au Conservatoire ou dans une École d'Art dramatique: par exemple celle du TNS à Strasbourg ou le CNSAD à Paris. Or le niveau des concours d'entrée est très élevé: 2000 à 7000 candidats pour 20 places. Je me suis aussi rendue compte que je n'adhère pas à ce que représente le théâtre français. J'aime assister à des pièces de théâtre français mais je n'aime pas le jeu des Français. Je suis plutôt habituée au théâtre russe. Sur scène, j'aime le naturalisme, la sincérité des émotions. À Chişinău, on enseignait un mélange du système Stanislavski, Mikhail Tchékov (le neveu de Anton Tchékov) - il s'agit d'un acteur, metteur en scène connu partout dans le milieu théâtral - et Vachtangov, un autre élève de Stanislavski. Les professeurs de mon lycée étaient des acteurs moldaves qui avaient fait leurs études en Russie, à Moscou, au MHAT (Théâtre d'Art de Moscou comportant une école associée à ce théâtre). D’autres professeurs avaient obtenu des licences en Russie et des masters professionnels en Moldavie.

H. L.: Certains réalisateurs moldaves se sont fait connaître à l'étranger, avec des films assez marqués, c'est vrai. Je pense à "Les Tziganes montent au ciel» 
inspiré de Maxime Gorki, un film soviétique de Emil Loteanu sorti en 1976. Avez-vous pensé ou imaginé faire du cinéma?

O.B.: Au départ, je ne l'avais pas envisagé. C'est le théâtre qui m’attirait. J'ai toujours pensé que mieux vaut jouer deux heures au théâtre face à un public que d'apparaître quelques minutes ou moins encore après avoir répété longtemps une réplique minuscule. Au théâtre, chaque moment est unique. Au cinéma, il faut produire une expression qui quand elle est saisie par la caméra devient déjà un cliché, une chose figée (roumain : şablon). Sur scène, je peux choisir d'être qui je veux - chaque fois que je monte sur un plateau de théâtre, je suis quelqu'un de différent. J'aime aussi le processus de création d'un rôle. C'est un peu cela qui est en ouvre dans le fait d'écrire des poèmes, des textes en prose, de les publier.

H. L.: Aimeriez-vous lire face à un public vos propres poèmes publiés?

O.B.: Oui! Moi: Olga actrice lisant les textes de Olga auteur, oui!

H.L.: Si vous ne connaissez pas personnellement de poètes de votre âge, êtesvous attentive à l'éventuelle activité d'auteurs de Moldaves de votre âge?

O. B.: Je peux vous parler d'une fille moldave de vingt ans aujourd'hui qui a publié en 2009, âgée de 18 ans un long poème qu'elle a traduit elle-même en français et anglais sur la Révolution moldave de 2009. Un de texte de mon livre s'en inspire. Les gens, la presse appellent cela «La Révolution Twitter» : il y a eu des morts, des gens torturés par la police. Tout le monde en parle en Moldavie mais il n'y a jamais eu de procès pour découvrir les coupables. Cette fille s'appelle Maria Paula Erizanu et son livre a pour titre: Aceasta este revolutia mea. Furați-mi-o!/ Ceci est ma Révolution. Volez-la moi! Le livre est une édition trilingue roumaine, française, anglaise, il compte une soixantaine de pages; tantôt les vers sont très brefs, tantôt il y a de vraies phrases. Cette fille est étudiante en philosophie à Londres. Elle a une plateforme online, un site qu'elle a créé. Elle a des amis écrivains, bloggers, journalistes. Je la vois souvent dans les «milieux jeunes» en Moldavie. On voit qu'elle s'est exprimée etc.

\section{H.L.: Existe-t-il des revues littéraires en Moldavie?}

O. B.: Oui un article sur mon livre signé Lidia Ungureanu a été publié dans Florile dalbe / Blanches fleurs une publication pour enfants et adolescents et dans Literatura şi arta / Art et Littérature, la revue culturelle et littéraire des écrivains moldaves. Un autre signé Iulian Filip a paru avant la publication de l'ouvrage dans une revue littéraire roumaine. Il contenait trois à quatre 
pages de bibliographie et des poèmes de Olga Baltag (avant qu'ils ne soient « rédactés» par Lidia Ungureanu qui est écrivaine).

H.L.: Seriez-vous d'accord pour que je traduise pour "Écrire ailleurs: deux Moldavies" - avec relecture par vous-même - trois textes qui m'ont beaucoup intéressée? Il s'agit de: "Iubea ploia / Elle aimait la pluie» qui évoque les effets d'une pluie radioactive - suite à Tchernobyl ou à Fukushima -, "Mergînd pe străzile Strasburgului/ Marchant dans les rues de Strasbourg» qui évoque la vie en France et "Mamă, tată, vă iubesc/Maman, Papa, je vous aime».

O.B.: Oui. Et d'ailleurs en ce moment, j'écris une pièce sur le thème de la Révolution qui fait l'objet du poème de Maria-Paula Erizanu.

\section{Mardi 20 novembre 2012}

H.L.: Quand j'ai vu la couverture de votre livre, le titre Autopsia sufletului m'a rappelé un titre de la romancière et essayiste française d'origine canadienne Nancy Huston: Autopsie de l'exil. Avez-vous entendu parler de Nancy Huston, Tzvetan Todorov?

O.B.: Je n'ai jamais entendu prononcer ces noms. Mon titre signifie simplement que je me livre au lecteur à nu. Par ces poèmes, je retire moimême les parties supérieures de mon âme face au lecteur pour que - au bout du compte - il me découvre telle que je suis: sincère, pure. Il s'agit d'une exposition.

H.L.: Je trouve vos poèmes abstraits, presque sans ancrage: lieu, temps, faits, anecdotes. Est-ce voulu?

O. B. : Certains poèmes s'adressent à des personnes bien précises. Au moment de la rédaction, avant de les publier surtout, j'ai modifié le côté personnel pour que le lecteur puisse s'identifier. En même temps, je constate que les personnes à qui s'adressaient ces poèmes se reconnaissent toujours (ils me disent: "ça c'est écrit sur moi ») alors que d'autres lecteurs imaginent que je parle d'eux. C'est ce que je voulais.

H.L.: Avez-vous l'impression que votre propos est tributaire de modes de communication Facebook, Twitter etc.

O.B.: Non en partie: je suis quelqu'un de traditionnel même si je suis née en 1990. Les croquis, fragments de textes qui me viennent en tête, je préfère les écrire sur papier ou envoyer une lettre à un ami que m'exprimer sur Facebook. 
Oui en partie: parce qu'on est dépendant des réseaux sociaux. Mais on n'apprend pas les nouveautés dans un magazine ou sur internet. J'aime la beauté du langage traditionnel. Certains de mes poèmes sont en anglais mais je n'aime pas introduire des mots anglais dans des poèmes en roumain par exemple. Je peux accepter les néologismes mais je préfère ne pas utiliser de jargon ou des abréviations, des sigles...

H.L.: Y a-t-il dans vos réserves beaucoup de poèmes que vous n'avez pas publiés?

O.B.: Certains textes sont trop personnels pour que j'aie eu envie de les publier. Quand je serai plus âgée, j'aimerais faire un recueil de lettres. J'aime bien correspondre avec mes amis qui sont en Moldavie ou ailleurs. Cela me donne la possibilité d'avoir entre les mains des mots pour toujours imprimés du papier. L'éternité des mots est très importante pour moi.

H. L.: De quoi parlent ces lettres?

O.B.: Ce sont des amis avec qui je parle tous les jours mais par lettres on se dit des choses qu'on n'oserait pas se dire oralement ou sur internet.

H.L.: Fréquentez-vous aussi des Roumains de Roumanie, êtes-vous allée souvent en Roumanie?

O. B.: Quand je vivais chez mes parents, on allait à la mer en Roumanie, à Constanța. Une fois quand j'avais 4 ans, on a campé: on était venus en voiture. Les tentes étaient plantées sur un promontoire très abrupt qu'il fallait descendre pour aller à la plage. On allait aussi à la plage ou à la mer en Ukraine, à Odessa qui a autrefois fait partie de la Roumanie. On y allait même plus souvent qu'en Roumanie. Quand j'avais 13-14 ans, ma troupe de théâtre est allée donner des représentations à Târgu-Neamț (Moldavie roumaine) sur l'invitation de l'arrière petite-fille de Ion Creangă: Olguța Caia. Donc, on a donné des représentations de Caragiale (premier grand dramaturge roumain du $\mathrm{XIX}^{\mathrm{e}}$ siècle considéré souvent comme tel jusqu'à nos jours), on a visité la maison de Creangă à Humuleşti, on a joué aux devinettes avec des gens (nous racontions des fragments d'histoires écrites par Creangă et les Roumains devaient situer le fragment). Nous étions logés dans les familles roumaines des enfants du chœur de l'église.

H.L.: Votre famille est-elle orthodoxe?

O. B.: Oui.

H.L.: Avez-vous été élevée dans la religion orthodoxe? 
O. B.: Oui mais je me suis rebellée.

H.L.: Connaissez-vous Savatie Bastovoï comme auteur?

O. B.: Un peu, j'ai commencé à lire "Les lapins ne meurent pas» parce que vous avez parlé de lui en cours.

H.L.: Y a-t-il d'autres auteurs moldaves ayant écrit en Roumanie et en roumain qui vous intéressent?

O. B. : ...

\section{Mardi 27 novembre 2012}

H. L.: Voulez-vous que nous parlions aujourd'hui de votre rapport d'auteur ou de personne à la langue roumaine, à la langue russe? Vous considérez-vous d'origine roumaine?

O. B.: Certains Moldaves «n'adhèrent pasà l'origine roumaine». Du côté de mon père, mes grands-parents sont nés en Grande Roumanie, mon grand-père a participé à la deuxième guerre mondiale du côté roumain. Il était paysan, il parlait le roumain dans sa famille, il a suivi sept classes primaires dans une école roumaine. Du côté maternel, mes grands-parents sont nés dans la RASSM : la République Autonome Soviétique Socialiste de Moldavie actuellement Transnistrie qui n'est reconnue que par la Russie et qui était plutôt peuplée par des Ukrainiens; les parents de ma mère étaient des intellectuels.

H.L.: Vous êtes née en 1990. Avez-vous toujours parlé roumain avec vos parents?

O. B.: Oui.

H.L.: A quel âge avez-vous appris le russe?

O.B.: Quand je sortais de la maison, je parlais russe avec des enfants du quartier qui parlaient russe. Je suis allée à 3 ans dans un jardin d'enfants où on parlait roumain. J'ai appris le russe à l'école comme matière obligatoire entre 10 et 11 ans: nous avions deux heures de russe par semaine.

H.L.: Vous considérez-vous comme bilingue russe-roumain?

O. B. : Oui.

H.L.: Avez-vous voyagé en Russie? 
O. B. : Non, jamais et je ne le regrette pas. Odessa, oui - où on parlait russe dans la rue et partout en raison de nombreux touristes est en Ukraine. Je ne suis jamais allée même en Crimée.

\section{H.L.: Quels auteurs lisiez-vous quand vous étiez enfant?}

O.B.: Je travaillais le français avec ma mère, professeur de français comme ma grand-mère. Elle m’apprenait les poésies qu'elle enseignait à ses élèves. Je connaissais des contes roumains depuis que j'étais toute petite. Ainsi "Capra cu trei iezi/ La chèvre et les trois chevreaux». Peut-être me le racontait-on au moment de m'endormir ou comme éducation orale. J'avais des livres de Creangă: Amintiri din copilărie/ Souvenirs d'enfance. J'ai encore un livre que j'ai colorié au stylo: il contenait des "contes universels » : dix contes roumains et des contes chinois, persans etc. Vers treize ans, j'ai lu Baltagul/Le Hachereau de Sadoveanu parce que tout le monde me demandait si je connaissais au moins la signification de mon patronyme: «baltag»= hachereau... Ce livre m’a profondément marquée: surtout le personnage de la femme Victoria Lipan qui cherche son mari disparu etc., puis qui veut venger sa mort. J'ai aimé aussi un livre de Aureliu Busuioc, un auteur moldave publié en Roumanie. Il avait pour titre Singur în fata dragostei/Seul face à l'amour. J'avais 14 ans, cette histoire d'amour m'a plu. À l'école, on apprenait des poésies du classique Mihai Eminescu (Xıx ${ }^{\mathrm{e}}$ siècle) que j'ai lu bien avant l'âge de 17 ans. On l'a étudié chaque année aussi dans le cadre des cours de roumain. Vers 17 ans j'en ai eu vraiment assez. Je me suis mise à lire des poètes comme Minulescu ou Bacovia, Mircea Cărtărescu. Je trouvais leurs textes dans les livres de roumain que je parcourais à l'avance avant qu'ils ne soient traités en classe. J'ai lu aussi certains de ces auteurs autrement qu'en extraits choisis pendant les activités extra-scolaires animées par la prof de théâtre qui était aussi notre professeur en classe puisque dès l'âge de 10 ans, je suivais les cours du lycée théâtral. C'est avec elle que nous avons fait ce grand voyage. En général, les élèves de ce lycée lisaient surtout des auteurs moldaves: Dumitru Matcovschi, Claudia Partole, Galina Martea, Grigore Vieru et aussi Ana Blandiana qui est roumaine.

H.L.: Je vous ai demandé lors de notre dernier entretien si vous connaissiez Leonida Lari qui a une ouvre poétique importante et qui a joué un rôle parlementaire. J'ai découvert sur Internet qu'à l'occasion de son décès, des adolescents moldaves étaient venus déposer une gerbe devant sa tombe. On avait l'impression d'une manifestation spontanée. 
O.B. (rires gênés): Elle a joué un rôle important dans le mouvement d'émancipation nationale en Moldavie entre 1988 et 1991. Elle était poète et publiciste aussi. Mais on ne parlait jamais d'elle à l'école. Elle a toujours voulu la réunion de la Moldavie et de la Roumanie. Lorsqu'en 1991, la Mare Adunare Națională/ Grande Assemblée Nationale à Chişinău réunie pour déclarer l'indépendance a demandé que le roumain soit langue officielle en Moldavie, mon père qui participait à ce mouvement de renaissance nationale m'a montré des photos (prouvant qu'il participait à ces manifestations). Il en a été ainsi mais par la suite, dans la foulée des émotions, le premier président élu de Moldavie a voulu demander l'Union avec la Roumanie sur la base du fait qu'une partie de la Moldavie était déjà roumaine et que nous, les Moldaves nous étions aussi des Roumains (comme le prouve le passé historique et politique à partir du XIX ${ }^{e}$ siècle). Du coup, au cours des années qui ont suivi dans ma famille on discutait beaucoup ces sujets. Mais les esprits se sont vite " calmés» sur intervention de la Russie naturellement. En 1992, pour en revenir à Leonida Lari, elle a été élue au Parlement de Roumanie comme député, sur les listes de România Mare/La grande Roumanie, le Parti nationaliste de droite qui a changé de nom. Elle est restée députée jusqu'en 2008.

H.L.: Avez-vous lu son ceuvre?

O.B.: Quelques petits poèmes très nostalgiques marqués d'un fort esprit patriotique qui me rappellent des débats avec mon père: pays, patrie etc. Olga lit un passage de Leonida Lari (proposition de traduction H. Lenz):

Nici harta nu-i ca alte dăți Même la carte n'est plus comme autrefois...

Din Nistru până-n Tisa

Moldova-i astăzi jumătăți

Cât rău făcutu-ni-s-a

Du Prut jusqu'à la Tisa

La Moldavie n'est plus que la moitié de ce qu'elle était Quel mal on nous a fait!

H.L.: Ces vers ne sont-ils pas des calques du poème d'Eminescu anti-russe et qui je crois bien a consacré sa réputation d'antisémite aujourd'hui remise en cause ou plutôt réhabilitée par la philosophe roumaine Marta Petreu? Le poème d'Eminescu aussi a cette formule: "De la Nistru până la Tisa»...

O. B.: Oui je trouve ces vers de Leonida Lari très eminesciens. Généralement, il y a en Moldavie aussi un culte d'Eminescu «Le grand poète roumain de tous les temps». C'est une honte de ne pas connaître au moins une strophe de Luceafàrul/ Hyperion qu'il faut savoir par cœur dès l'âge de 10 ans (on l'apprend tous les ans à l'école). Tout le monde en Moldavie connaît au moins les premières strophes de ce poème. 


\section{Mardi 11 décembre 2012}

H.L.: Si vous l'aviez souhaité, auriez-vous pu faire des études en Roumanie? Les Universités roumaines accueillent-elles les Moldaves?

O.B.: Chaque année, 5000 bourses sont accordées à des Moldaves par le gouvernement roumain dans tous les domaines d'études. Certains partent faire des études en Roumanie sans bourses même. Car pour les Moldaves, les études ne sont pas payantes: il n'y a pas de taxes d'inscription (pour un étudiant roumain les taxes fluctuent entre 1000 et 6000 lei roumains). La monnaie moldave est aussi le LEU sans doute mais le taux de change n'est pas égal. Un leu roumain équivaut à 4 lei moldaves. En 2009, quand j'ai reçu la lettre confirmant mon acceptation à l'Université de Strasbourg, j'ai déposé ma candidature en Roumanie aussi en vue de faire des études à Cluj-Napoca ou à Bucarest. Mais malheureusement (ou peut-être heureusement), je n'ai pas été acceptée. Du coup, je suis venue faire mes études en France. 